\title{
Reducing GPS carrier phase errors in the measurement and position domains for short-distance static relative positioning
}

\author{
Chao Liu ${ }^{1} \cdot$ Peng Lin $^{1} \cdot$ Xingwang Zhao $^{1} \cdot$ Jingxiang Gao $^{2}$
}

Received: 23 September 2014/Accepted: 3 April 2015/Published online: 19 April 2015

(C) Akadémiai Kiadó 2015

\begin{abstract}
In GPS short-distance static relative positioning (e.g. the length of the baseline shorter than several kilometers and its height difference less than tens of meters), the precision and accuracy of the baseline solutions are limited because of the existence of unmodeled errors especially multipath errors. Considering that multipath errors are mapped from original observations to the baseline solution (position domain) and its residual series (measurement domain), scholars have applied a number of methods to reduce multipath errors in residual series to improve the precision of the baseline solution. However, multipath errors are difficult to eliminate from the baseline solution. Therefore, this study proposes a new strategy for eliminating the multipath errors both in the position and measurement domains. The steps are as follows: (1) a post-processed kinematic (PPK) approach was used to obtain the coordinate and residual series with $\mathrm{L}_{1}$ observations; (2) the empirical mode decomposition (EMD) was employed to extract multipath errors from the residual series to correct original observations; (3) PPK was used again to obtain the corrected coordinate series with the corrected observations; (4) finally, a multi-scale decomposition utilizing wavelet transform was applied to the corrected coordinate series to obtain the residual terms and their average values, the result of which is taken as the final baseline solution. The precision and accuracy of the baseline solution are evaluated according to the root mean square error (RMSE). The experimental results show that the accuracy of baseline solution are improved about 16, 24, and $28 \%$ for $10 \mathrm{~min}$ time periods, respectively, 24, 25, and $33 \%$ for 30 min time periods, respectively, and 5, 23, and $22 \%$ for 60 min time periods, respectively, of the traditional scheme. The influence of multipath errors on the baseline solution are efficiently weakened.
\end{abstract}

Chao Liu

chaoliu0202@gmail.com

1 School of Geodesy \& Geomatics, Anhui University of Science \& Technology, Huainan 232001, China

2 School of Environment \& Spatial Informatics, China University of Mining \& Technology, Xuzhou 221116, China 
Keywords GPS - Baseline solution - Multipath errors - Static relative positioning $\cdot$ Postprocessed kinematic (PPK)

\section{Introduction}

GPS static relative positioning is jointly affected by orbit errors, ionospheric delay, tropospheric delay, antenna phase center deviation, and multipath errors (Xu 2007; Hastaoglu and Sanli 2011). For short-distance static relative positioning with the distance of the two sites within several kilometers and its height difference less than tens of meters, the double-difference technique can eliminate or basically limit the influence of distancedependent errors, such as ionospheric and tropospheric delays, and orbit errors. However, site-dependent multipath errors occur when signals are reflected off nearby objects, such as water surfaces, trees, buildings and so on, and received together with the direct signals (Dai et al. 2014). Thus, multipath errors and the potential diffraction errors become the major error sources which affect the precision and accuracy of GPS relative positioning because it cannot be effectively eliminated by the double-difference technique, especially under short observation times and large sampling intervals (Tut et al. 2013).

Designing excellent receiving antennas can significantly reduce the influence of multipath signals (Townsend et al. 1995; Boccia et al. 2014). However, multipath signals cannot be completely suppressed while receiving effective signals (Stewart et al. 2005). Therefore, the multipath errors will continue to be the main error source of GPS relative positioning in the foreseeable future. This problem remains significant for geodesists who wish to establish a rigorous model for parameter estimation. Existing studies mostly focus on optimizing both stochastic and functional models to eliminate or reduce the influence of multipath errors on parameter estimation.

The influence of multipath errors on parameter estimation can be reduced, such that the positioning precision can be improved by optimizing the stochastic model for GPS relative positioning. Given that the quality of observations differs at different observation stations, satellites and epochs, the appropriate stochastic model should be determined when the least square method is used for parameter estimation (Xu 2007). Observations are generally believed to be mutually independent and with equivalent precision, i.e., the equal-weighted stochastic model, which is obviously impractical. When multipath and diffraction errors are large, the baseline solution obtained through the equal-weighted stochastic model deviate to a certain extent. An ideal stochastic model should enable to establish a realistic variance-covariance information of observations that can accurately reflect the quality of observations. A stochastic model based on satellite elevation angle, signal-to-noise ratio or signal intensity is an approximation of the ideal stochastic model. In models based on satellite elevation angle, the relationship between the observation quality of the carrier phase signal and the elevation angle can be used to obtain the variance-covariance of observations (Wang et al. 1998). Models based on signal-to-noise ratio and signal intensity represent the variance-covariance of observations using the observation quality of carrier phase signals (Hartinger and Brunner 1999; Brunner et al. 1999; Dai et al. 2008a). Furthermore, variance component estimation is a rigorous method for estimating the variancecovariance of observations. By using an iterative algorithm, we can estimate the unit weight variance for different observations that are influenced by various factors. This method has been applied to estimate the variance-covariance of GPS carrier phase 
observations, and ideal results have been achieved (Wang et al. 2002; Satirapod et al. 2002; Satirapod 2006). Although the stochastic models can weaken the influence of the multipath errors on baseline solutions to various extents, the multipath errors are not eliminated (or reduced) at all. Instead, smaller weights are rendered for observations with poor quality. The systematic and regular nature of multipath errors require the further improvement of functional models.

By improving the functional model for GPS relative positioning, multipath errors can be reduced, and positioning precision can be increased. Given that the spatial correlation of the multipath errors is rapidly attenuated with the movement of the observation stations and that the observation environment is complex and variable, establishing a model for multipath errors in the absence of prior information is difficult (Dai et al. 2008b). Given that the residual series can reflect the quality of observation signals to some extent, multipath errors in residual series can be extracted using certain methods to correct the original observations. Thus, the baseline solution, which reduces the multipath errors to a certain extent and increases the precision of the baseline solution, can again be performed with the corrected observations (Satirapod and Rizos 2005; Wang et al. 2008, 2009; Liu et al. 2010; Azarbad and Mosavi 2014).

However, in the baseline solution process, multipath of the original observations is mapped to the baseline solution (position domain) and its residual series (measurement domain). If only the multipath errors in the measurement domain are extracted and removed, the precision of the baseline solution can be increased. However, the multipath errors in the position domain are not weakened and are thus difficult to process directly. Further studies should be conducted to eliminate the multipath errors in the position domain and to determine its influence on the precision and accuracy of baseline solutions. In this study we propose the post-processed kinematic approach (PPK) instead of the traditional method for static relative positioning. Moreover, the coordinate series and its residual series are obtained. Subsequently, empirical mode decomposition (EMD) and wavelet transform are employed to eliminate multipath errors both in the position and measurement domains. Thus, the precision and accuracy of baseline solutions can theoretically be increased.

The remainder of this paper is organized as follows: Sect. 2 provides the new scheme of baseline solutions and the data process procedures. Sections 3 and 4 present and compare the experimental results of baseline solutions using different schemes. Section 5 summarizes the contents of this article.

\section{GPS baseline solution scheme based on the reduction of unmodeled errors in both the measurement and position domains}

The functional model for short-distance GPS carrier phase relative positioning is expressed as follows (Wang et al. 2009):

$$
\lambda \cdot \nabla \Delta L=\nabla \Delta \rho-\lambda \cdot \nabla \Delta N+\nabla \Delta V_{\text {multipath }}+\nabla \Delta V_{\sigma}
$$

where $\nabla \Delta$ is the double-difference operator; $\lambda$ is the wavelength; $\nabla \Delta L$ denotes the doubledifference carrier phase observations; $\nabla \Delta \rho$ is the double-difference distance between the satellites and the receivers; $\nabla \Delta N$ are the double-difference integer ambiguities; $\nabla \Delta V_{\text {multipath }}$ is the multipath error; and $\nabla \Delta V_{\sigma}$ expresses the noise. The influence of orbit 
errors, troposphere delay and ionosphere delay are ignored in Eq. (1) for short-distance and small height difference of the baseline.

To characterize the basic influence of multipath, we suppose only one multipath signal exists and a simple multi-ray signal model can be used. A direct signal $\mathrm{S}_{1}$ can be written as follows:

$$
\mathrm{S}_{1}=\mathrm{A} \sin \omega t
$$

where $\mathrm{A}$ is the signal amplitude; $\omega$ is the angular frequency. Using the geometry relation, a reflected signal $\mathrm{S}_{2}$ can be obtained:

$$
\mathrm{S}_{2}=\alpha_{1} \mathrm{~A} \sin (\omega t+\Delta)
$$

where $\alpha_{1}$ is the attenuation coefficient; $\Delta$ is the phase shift between the direct and the reflected signals. Then, the multipath error $\mathrm{S}$ is:

$$
\mathrm{S}=\alpha_{0} \mathrm{~A} \sin \left(\omega t+\Delta_{0}\right)
$$

where $\alpha_{0}=\sqrt{1+2 \alpha_{1} \cos \Delta+\alpha_{1}^{2}}, \Delta_{0}=\arctan \left(\frac{a_{1} \sin \Delta}{1+a_{1} \cos \Delta}\right)$. The theoretical maximum of the multipath error is $0.25 \lambda$ while $\alpha_{1}=1$ and $\Delta=\frac{\pi}{2}$. This corresponds to $4.6 \mathrm{~cm}$ for GPS $\mathrm{L}_{1}$ carrier phase observation.

Owing to the existence of multipath errors, the baseline solution will show certain deviations, and the residual series will also contain some unmodeled errors. The scheme proposed in this article is used for data processing with the procedures shown in Fig. 1: (1) the original GPS observations are used to constitute the double-difference observation equation for PPK, and the coordinate and residual series are obtained; (2) the EMD method is employed to extract the multipath errors in the residual series to correct the original observations and to eliminate the influence of multipath errors in the measurement domain on the baseline solution; (3) the corrected observations are again used for PPK, and the corrected coordinate series is obtained; (4) the corrected coordinate series is used for

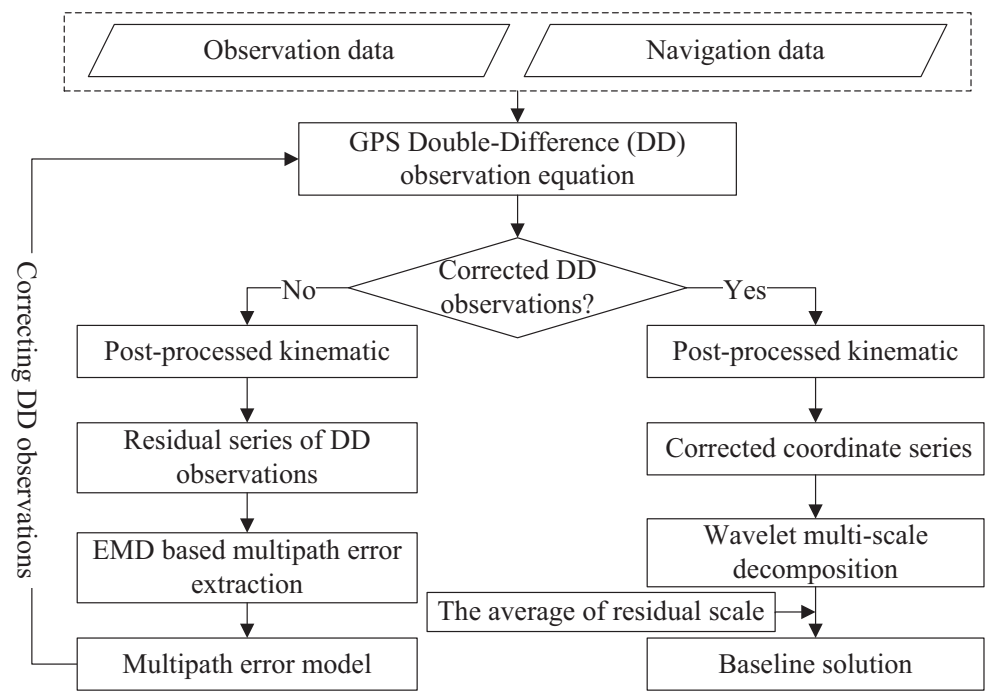

Fig. 1 Functional block diagram of the proposed GPS baseline solution scheme 
wavelet multi-scale decomposition, the residual scale is obtained, and the average is taken as the final baseline solution. After data processing, the multipath errors in the measurement domain can basically be removed. Moreover, the multipath errors in the position domain can be significantly reduced (only the low-frequency information on the residual scale is preserved through wavelet decomposition). Therefore, the precision and accuracy of short-distance GPS static relative positioning can be improved. The EMD and wavelet function model applied in this study are similar to those utilized by Wang et al. (2009).

The new method proposed in this article obtains the coordinate series instead of the baseline solution at the expense of redundant observations. When integer ambiguities are fixed, the traditional method for a static baseline solution is employed to obtain the redundant number of observations of $N_{\mathrm{e}} \cdot\left(N_{\mathrm{s}}-1\right)-3$, where $N_{\mathrm{e}}$ is the number of epochs, and $N_{\mathrm{s}}$ is the number of satellites for simultaneous observations in a certain epoch between the two stations. For the PPK, a baseline solution is necessary for each epoch, and the redundant observations are $N_{\mathrm{e}} \cdot\left(N_{\mathrm{s}}-1-3\right)$. If the number of satellites is 7 , the observation time is $30 \mathrm{~min}$, the sampling interval is $1 \mathrm{~s}$, the number of redundant observations for the static baseline solution is 10,797 , and the number of redundant observations for PPK is 5400. So the number of redundant observations is reduced to approximately its half. Thus, the reliability of parameter estimation may be reduced. However, this configuration has two advantages: (1) PPK can provide both the residual and coordinate series, which can more intuitively reflect multipath errors and possible gross errors. Thus, we can perform a corresponding treatment using certain methods to improve the accuracy and precision of the static baseline solution. (2) The gross errors probably present in different epochs do not influence other epochs under PPK. Moreover, gross errors are easier to discover and to eliminate.

Given the existence of multipath errors, the degree of density or discreteness of the estimated parameters (baseline solution) should be described along with the closeness to true values. At this time, the standard deviation is insufficient as the evaluation indicator. Thus, the root mean square error (RMSE) is introduced to represent the quality of parameters under the joint influence of random and systematic errors. The expression reads

$$
\operatorname{RMSE}(\boldsymbol{X})=\sqrt{\mathrm{E}(\boldsymbol{X}-\tilde{\boldsymbol{X}})^{2}}
$$

where $\boldsymbol{X}$ is the estimated parameter, and $\tilde{\boldsymbol{X}}$ is the true value of the parameter. When no systematic error is present, RMSE becomes the standard deviation.

\section{Data collection and processing}

\subsection{Data collection}

Data were collected during the period 1:30-9:20 UTC (local time 9:30-17:20) on March 28, 2009 (DOY 087, GPS week 1524), at two sites located at the campus of China

Table 1 Baseline vectors of experimental data/m

\begin{tabular}{llll}
\hline Direction & $\mathrm{N}$ & $\mathrm{E}$ & $\mathrm{U}$ \\
\hline Value & 175.5277 & 28.0917 & 39.4439 \\
\hline
\end{tabular}


University of Mining and Technology. Two Ashtech UZ-12 dual-frequency receivers were set up at two stations with a separation of approximately $182 \mathrm{~m}$ for data collection. The sampling interval was $1 \mathrm{~s}$, and the cut-off elevation angle was set as $10^{\circ}$. Table 1 provides the reference baseline vector components of the above observation using traditional static baseline solution method, which was taken as the true value. The coordinate series utilizing PPK is shown in Fig. 2. For convenience of calculation and analysis, only the coordinate series between local time 10:00-17:00 are displayed.

\subsection{Data processing}

Given the low-frequency feature of multipath errors compared to random errors, the performance of various baseline solution methods is evaluated under different observation periods. The observation data are segmented on the hour from 10:00 to 17:00 with the duration of 10, 30, and $60 \mathrm{~min}$ (Fig. 2). Seven time periods of 10, 30, and $60 \mathrm{~min}$ are selected for data processing and analysis.

The equal-weighted stochastic model is adopted for the baseline solution using four schemes. In Scheme 1, the traditional static baseline solution method is used. In Scheme 2, PPK is applied for the baseline solution, and the coordinate series is obtained. The average of the coordinate series is taken as the final baseline solution. Wang et al. (2009) proposed the model used in Scheme 3. The EMD-Wavelet model is used to extract multipath errors from the residual series of Scheme 1 and to correct the original observations. Then, the baseline solution is performed again with the corrected observations (Wang et al. 2009). In Scheme 4, the new model proposed in this article is used. Given that the theories for data processing in Schemes 1 and 2 are already mature, the data processing procedures are not described in detail. Details are given by $\mathrm{Xu}$ (2007). The data processing procedures in Scheme 3 are introduced in the literature (Wang et al. 2009). In this article, we only present the data processing procedures for Scheme 4.

Data processing and analysis are described for the first 10 min time period. Residual series are obtained by PPK in this period, and the EMD method is employed to extract the multipath errors. The extraction procedures are given in Fig. 3a, b, c, and d. Figure 3a presents the original residual series of six pairs of satellites: PRN02-04, PRN02-24,

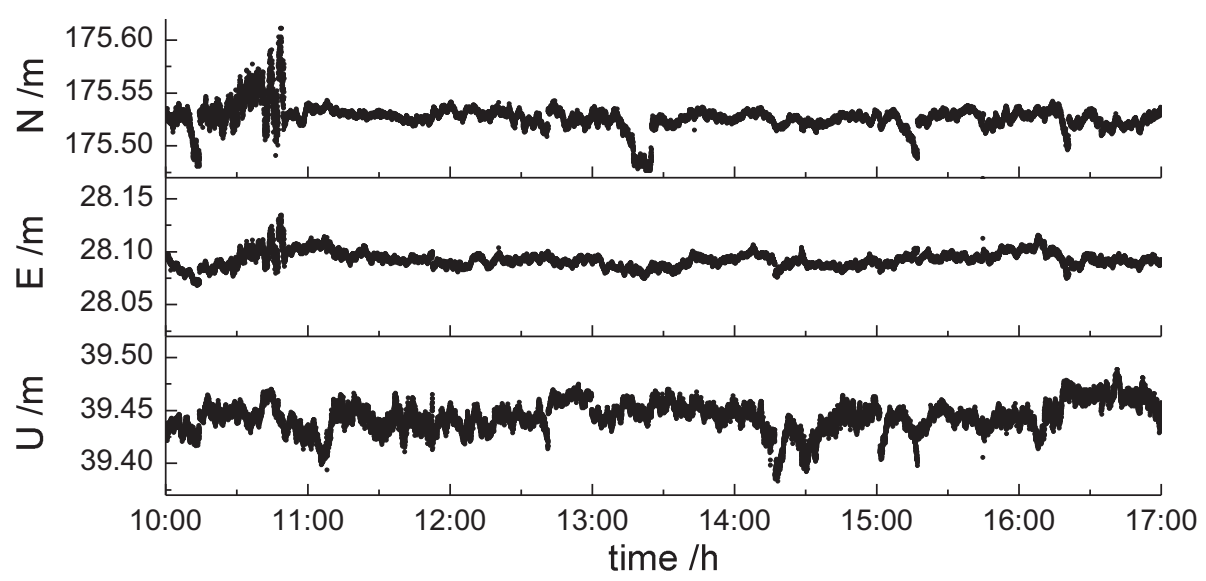

Fig. 2 Baseline vector components time series 
(a)

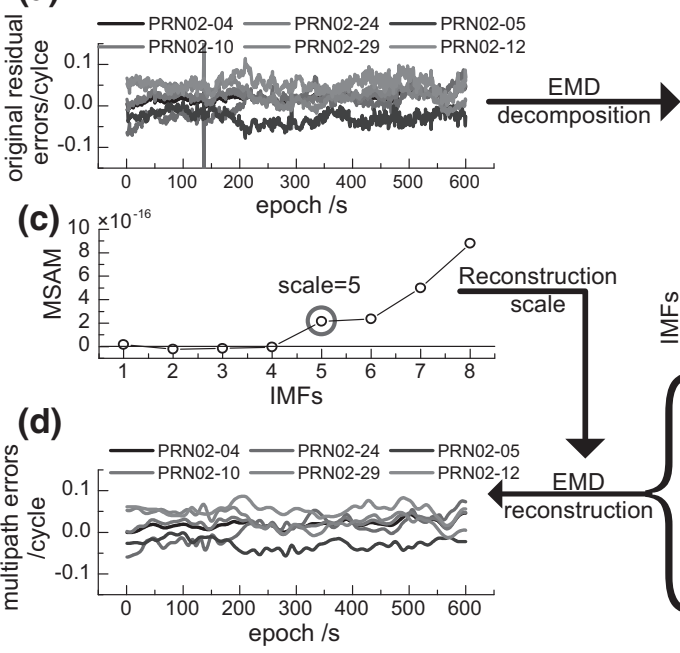

(b)

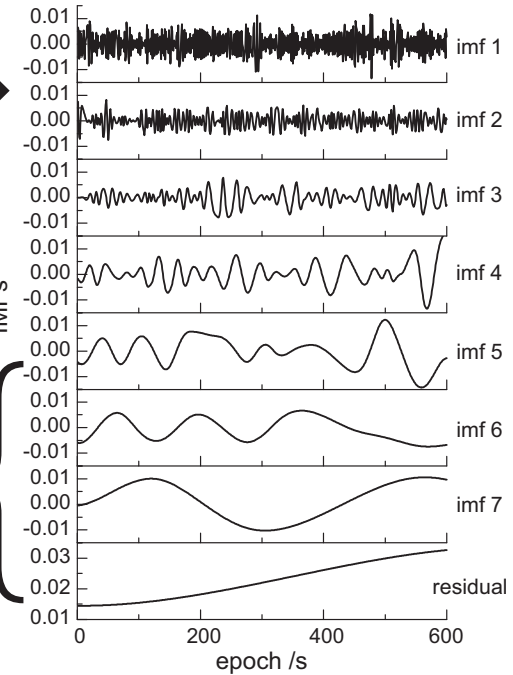

Fig. 3 Procedures for the extraction of multipath errors in measurement domain using EMD method (the first 10 min time period). a Original residual series, b EMD decomposition results of residual series for PRN02-10, $\mathbf{c}$ relationship between the MSAM and the scale, $\mathbf{d}$ extracted multipath errors

PRN02-05, PRN02-10, PRN02-29, and PRN02-12. These series contain obvious unmodeled errors. A detailed illustration is given with PRN02-10 as an example. Figure $3 \mathrm{~b}$ shows the EMD decomposition results of residual series for PRN02-10. The figure has seven intrinsic mode functions (IMF) and one residual term. The relationship between the means of the accumulated standardized modes (MSAM) and the scale is given in Fig. 3c (Wang et al. 2009). The appropriate scale for the discrimination of high- and low-frequency IMF is 5. Each high-frequency IMF (from IMF1 to IMF4) is de-noised, and the EMD reconstruction gives the extracted multipath errors (from IMF5 to IMF7 and the residual term) in Fig. 3d. Another five multipath errors are extracted from the corresponding residual series for the remaining satellite pair using the same procedure (Fig. 3d).

The extracted multipath errors are used to correct the original observations, and PPK is again applied. The corrected coordinate series (Fig. 4a) and residual series (Fig. 4b) are

(a)

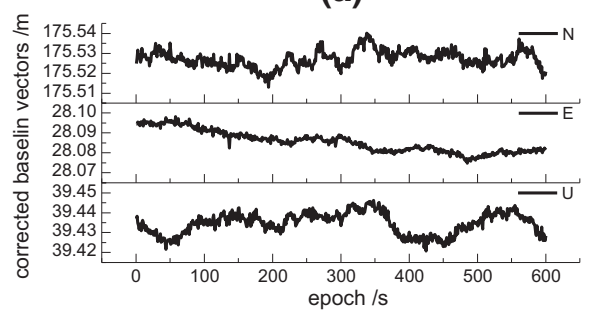

(b)

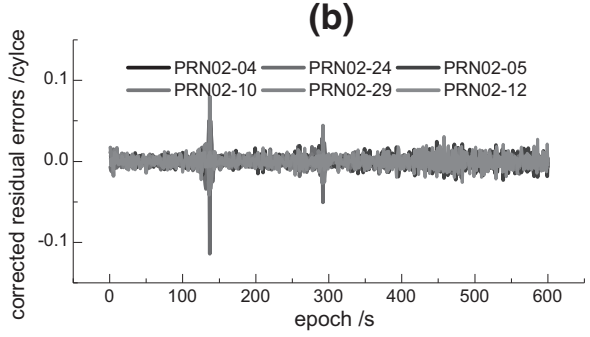

Fig. 4 Coordinate series and residual series with corrected observations. a Corrected coordinate series, b corrected residual series 
Signal
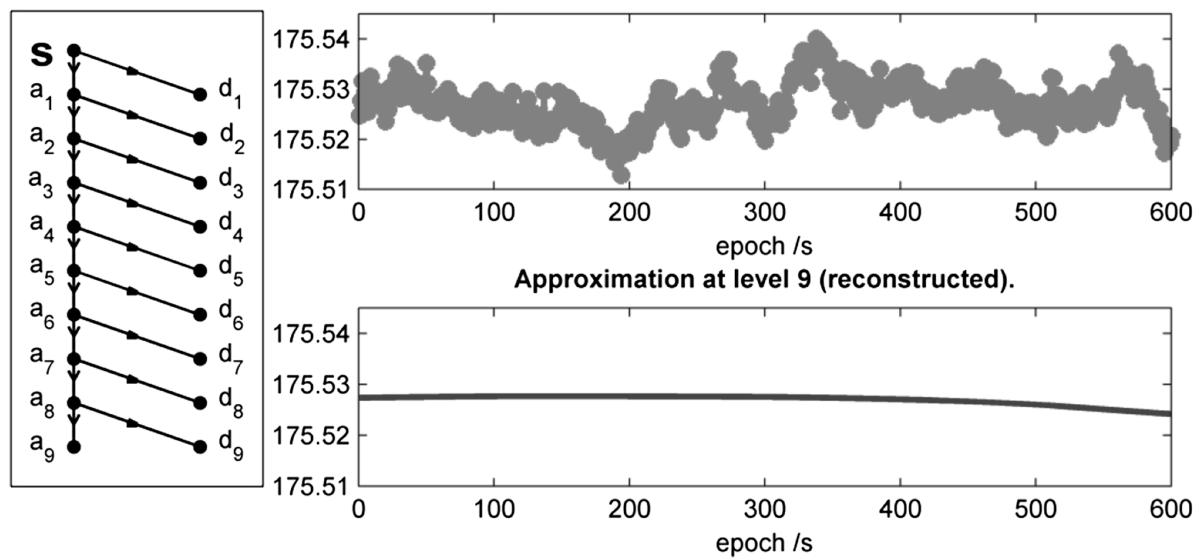

Fig. 5 Schematic of wavelet decomposition on coordinate series in $\mathrm{N}$ direction in the first 10 min period (the upper right is the original coordinate series; the left is the wavelet tree; the bottom right is the residual scale)

obtained. Compared with the original residual series (Fig. 3a), the corrected residual series shows a significant stochastic behavior. However, the new coordinate series still contain obvious unmodeled errors (Fig. 4a), and further processing is required.

Now the coordinate series in Fig. $4 \mathrm{a}$ is subject to wavelet decomposition. The $\mathrm{N}$ direction is taken with a residual scale of a9 (Fig. 5, bottom right). In Fig. 5, upper right, the "sym5" wavelet is selected, and the decomposition scale is 9. Thus, nine scales are obtained: d1, d2,..,d9 (Fig. 5, left). The average of residual scale a9 is taken as the final baseline solution in the $\mathrm{N}$ direction. The same process is applied to other directions and time periods. Figure 6 shows the baseline solutions obtained using four different solution schemes in all time periods of 10,30 and $60 \mathrm{~min}$. The green solid lines are the true values.

\section{Result analysis}

Figure 6 shows: (1) the four baseline solution schemes can yield consistent baseline solutions. As the observation time increases, the baseline solutions become closer to the true values. Thus, a prolonged observation time can reduce the influence of multipath errors on baseline solutions to a certain extent. (2) The baseline solutions of Schemes 1 and 2 are basically consistent within all seven 10 min time periods. The 30 and 60 min time periods occasionally exhibit some minor variations, but the results are basically consistent. Thus, using the average of coordinate series as the baseline solution is feasible. (3) In all time periods, the results of Schemes 1 and 3 maintain high consistency. Thus, Schemes 1 and 3 have comparable accuracy. (4) Compared with other schemes, Scheme 4 can yield baseline solutions that are closer to true values. Different schemes are compared in the following sections. 

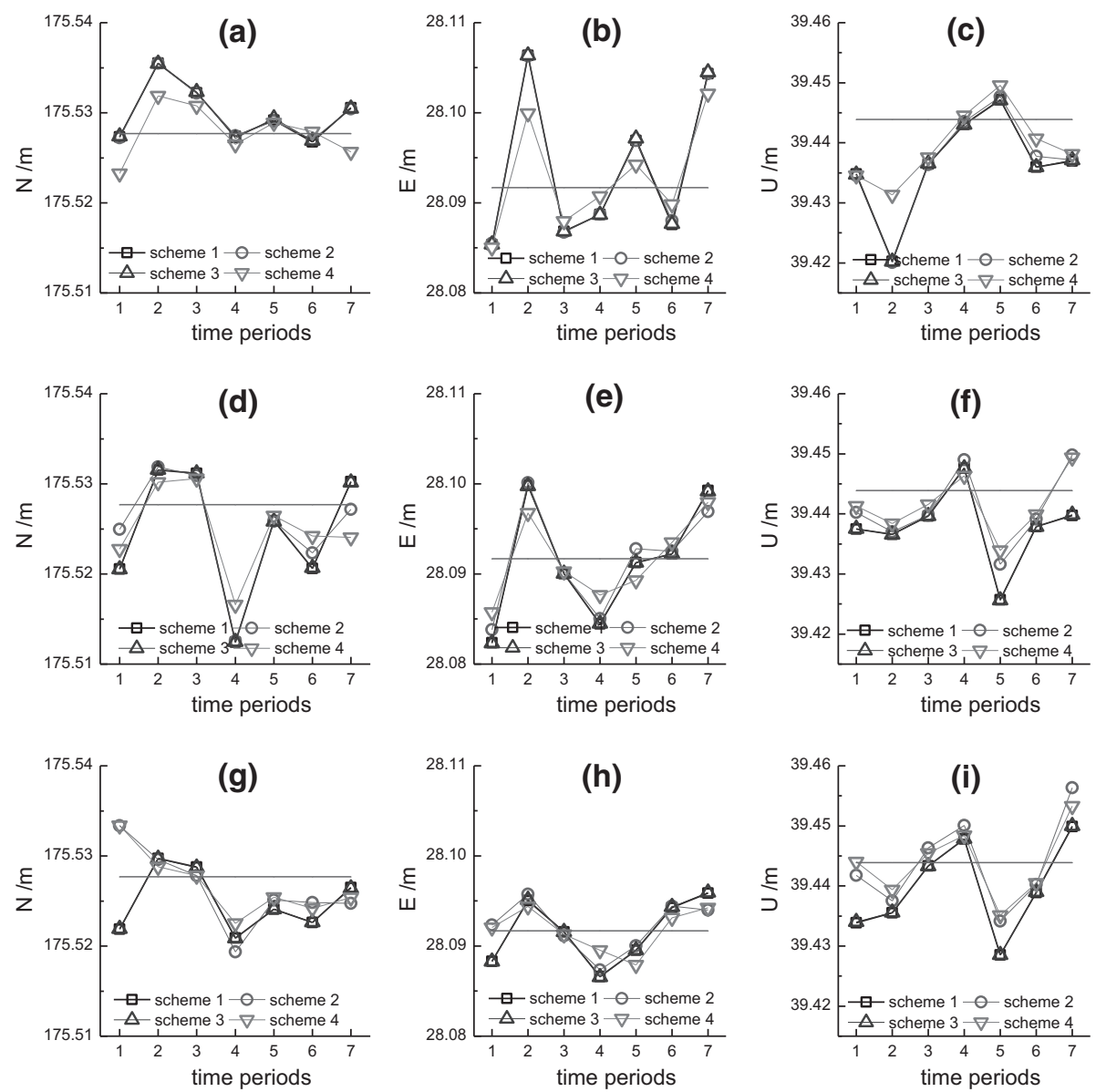

Fig. 6 Comparison of the results of four baseline solution schemes. a $10 \mathrm{~min}, \mathrm{~N}$ direction; b $10 \mathrm{~min}, \mathrm{E}$ direction; c $10 \mathrm{~min}$, U direction; d $30 \mathrm{~min}, \mathrm{~N}$ direction; e $30 \mathrm{~min}$, $\mathrm{E}$ direction; f $30 \mathrm{~min}$, U direction; g $60 \mathrm{~min}, \mathrm{~N}$ direction; h $60 \mathrm{~min}$, E direction; i $60 \mathrm{~min}$, U direction

\subsection{Comparison between Schemes 1 and 3}

The precision and accuracy of baseline solutions obtained by using Schemes 1 and 3 are compared and analyzed. Figure 7 shows the baseline solutions and the standard deviations obtained using these schemes. The $\mathrm{X}$ axis represents the time period; " 1 " represents the result of the first time period processed by Scheme 1; "2" represents the result of the second time period processed by Scheme 1, and so on. "E" represents the EMD-Wavelet in Scheme 3, "1E" is the first time period processed by Scheme 3, "2E" represents the result of the second time period processed by Scheme 3, and so forth. The black dots represent the baseline solution. The length of the line segment represents the magnitude of standard deviation of the baseline solution, and the green solid lines denote the reference value.

Figure 7 shows that for the same time period, the standard deviation of the baseline solution by Scheme 3 is significantly lower than that of Scheme 1. This method can 


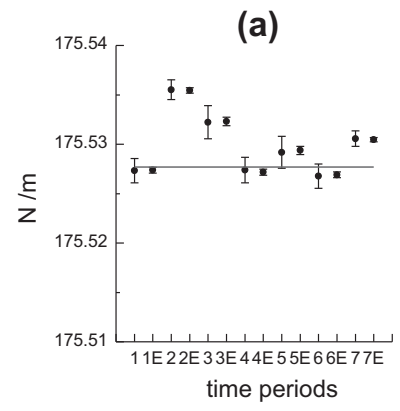

(b)
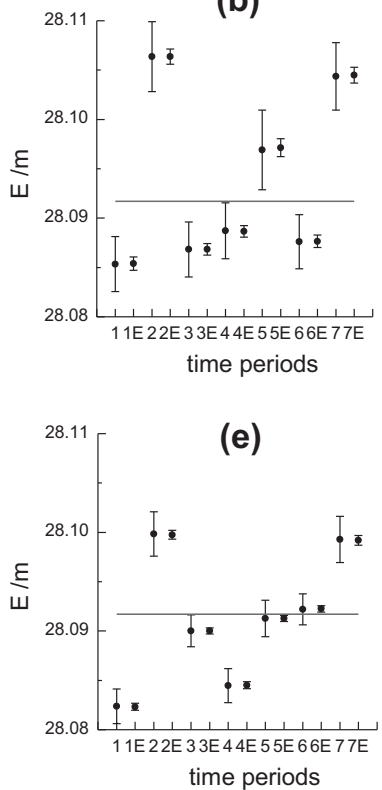

(h)

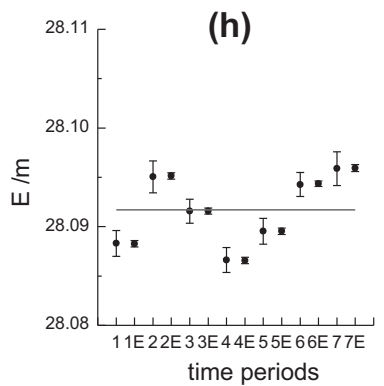

(c)

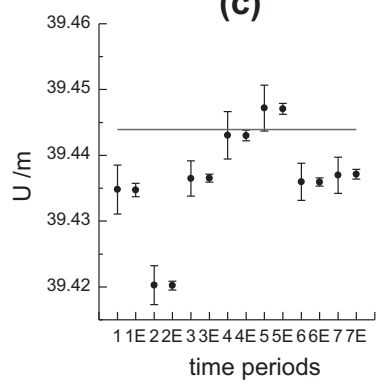

(f)

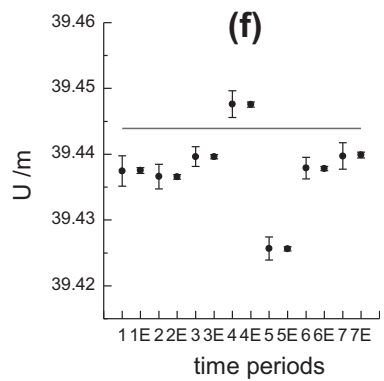

(i)

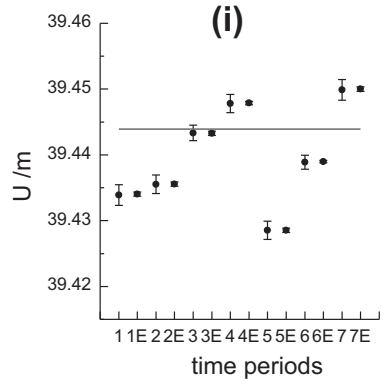

Fig. 7 Baseline solutions and standard deviation obtained using Schemes 1 and 3. a 10 min, $\mathrm{N}$ direction; b $10 \mathrm{~min}$, E direction; c $10 \mathrm{~min}$, U direction; d $30 \mathrm{~min}, \mathrm{~N}$ direction; e $30 \mathrm{~min}$, E direction; f $30 \mathrm{~min}$, U direction; g $60 \mathrm{~min}$, N direction; h $60 \mathrm{~min}$, E direction; i $60 \mathrm{~min}$, U direction

effectively improve the precision of the baseline solution, which is consistent with the conclusions reached in the literature (Wang et al. 2009). However, the baseline solutions obtained using Schemes 1 and 3 are not obviously different. For all the time periods in Fig. 7, the maximum difference between the baseline solutions obtained in three directions $(\mathrm{N}, \mathrm{E}, \mathrm{U})$ using the two schemes is $0.2 \mathrm{~mm}$. Therefore, compared with Scheme 1, Scheme 3 does not considerably improve the accuracy of the baseline solution.

To analyze the causes for the formation of the problem above, Fig. 8a presents the original residual series in the first $10 \mathrm{~min}$ time period. The results contain obvious unmodeled errors. The EMD-Wavelet model in Scheme 3 is used to extract and remove unmodeled errors. The remaining residual series is shown in Fig. 8b. Scheme 3 replaces the original residual series in Fig. 8a with that in Fig. 8b, and parameter estimation is again performed. The criterion for parameter estimation using the least square method is $\boldsymbol{V}^{\mathrm{T}} \boldsymbol{P} \boldsymbol{V}=\min$. However, the residual series in Fig. 8b shows strong 
(a)

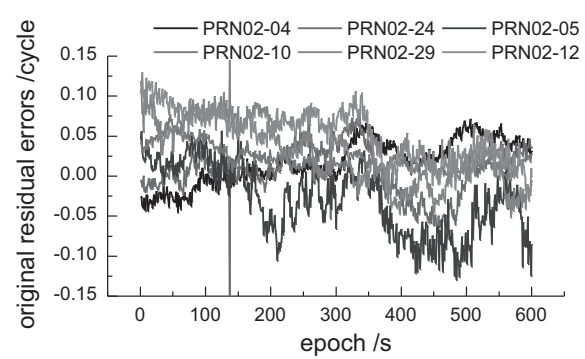

(b)

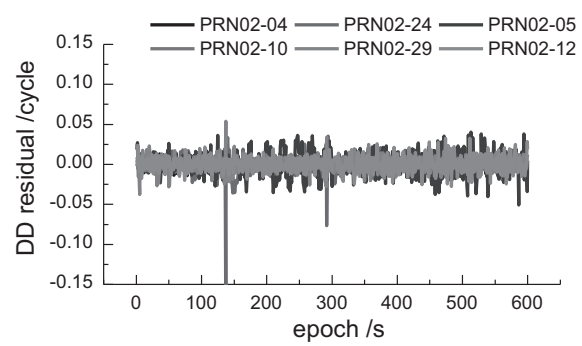

(c)

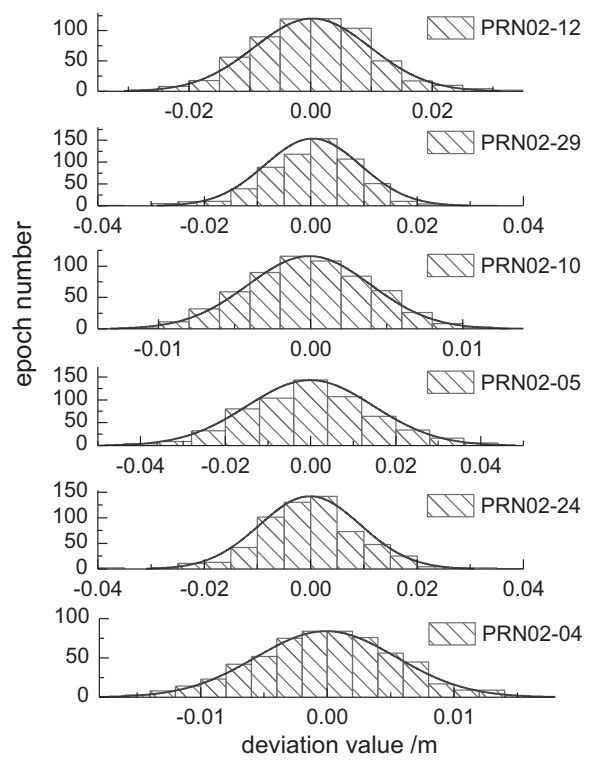

Fig. 8 Measurement domain analysis based on Scheme 3 (the first 10 min time period). a Original residual errors, $\mathbf{b}$ corrected residual errors, $\mathbf{c}$ histogram of corrected residual errors

Table 2 Root mean square errors obtained by four different schemes/mm

\begin{tabular}{llllll}
\hline Time length & Direction & Scheme 1 & Scheme 2 & Scheme 3 & Scheme 4 \\
\hline $10 \mathrm{~min}$ & $\mathrm{~N}$ & 3.2 & 3.2 & 3.2 & 2.7 \\
& $\mathrm{E}$ & 7.5 & 7.5 & 7.5 & 5.7 \\
\multirow{5}{*}{$30 \mathrm{~min}$} & $\mathrm{U}$ & 9.6 & 9.6 & 9.6 & 6.9 \\
& $\mathrm{~N}$ & 6.7 & 6.5 & 6.6 & 5.1 \\
& $\mathrm{E}$ & 5.6 & 5.5 & 5.6 & 4.2 \\
$60 \mathrm{~min}$ & $\mathrm{U}$ & 7.8 & 6.7 & 7.8 & 5.2 \\
& $\mathrm{~N}$ & 3.9 & 4.6 & 3.9 & 3.7 \\
& $\mathrm{E}$ & 3.1 & 2.9 & 3.1 & 2.4 \\
& $\mathrm{U}$ & 7.7 & 7.6 & 7.7 & 6.0 \\
\hline
\end{tabular}

stochasticity (the histogram corresponding to the series in Fig. 8b is shown in Fig. 8c). It can be predicted that when Scheme 3 is used for the baseline solution, the standard deviation of the parameter estimation will decrease significantly, whereas the estimated parameters are similar to Scheme 1. The RMSE of the baseline solution remains unchanged or varies only slightly. The baseline solution obtained using Scheme 3 remains affected by the same multipath errors similar to Scheme 1. If the standard deviation is used as the evaluation indicator, the quality of the parameter evaluation becomes difficult to assess. 


\subsection{Comparison between Schemes 3 and 4}

Unmodeled errors affect the baseline solutions obtained by different schemes to various extents. Therefore, the RMSE is used to evaluate baseline solutions. Table 2 shows the RMSE of baseline solutions obtained using the four different schemes in all time periods. The RMSE obtained using Scheme 4 is generally lower than those obtained by using the other three schemes. Thus, the precision and accuracy of baseline solutions are improved.

Regardless of the scheme used, the unmodeled errors in the original observations influence the baseline solutions and residual series. In Scheme 3, the traditional static baseline solution scheme is applied to obtain baseline solutions and residual series. However, only the multipath errors in the residual series can be extracted and weakened. The baseline solutions affected by multipath errors cannot be properly processed. Scheme 4 is the application of PPK to the baseline at the expense of redundant observations. Moreover, the residual and coordinate series are obtained. The multipath errors in residual and coordinate series are effectively reduced. The influence of multipath errors on the baseline solution is significantly reduced, and the precision and accuracy of the baseline solution are enhanced.

\section{Conclusions}

In the PPK positioning, the unmodeled errors in the observations will map to the residual and coordinate series. We propose two different filtering methods to reduce multipath errors in the two domains. Three other baseline solution schemes, including the one proposed by Wang et al. (2009), are compared. The results show that compared with the traditional static baseline solution scheme, the scheme proposed by Wang et al. (2009) can effectively increase the precision of the baseline solution, though the accuracy is barely improved. With the use of the scheme proposed in this study, the RMSE of baseline vectors are decreased by about 16, 24, and $28 \%$ for 10 min time periods, respectively, 24, 25, and $33 \%$ for 30 min time periods, respectively, and 5, 23, and $22 \%$ for 60 min time periods, respectively, of the traditional scheme. Nevertheless, the proposed scheme will increase the computational burden because of the additional course of the extraction of multipath errors in measurement and position domains and the baseline solution with the corrected observations. Compared to the traditional static baseline solution scheme, the increase of computation time is about a factor of 2. However, for a small- or median-scale GPS network, such as landslide monitoring network, mining subsidence monitoring network and so on, the computational effort is acceptable.

Acknowledgments The paper is sponsored by the National Natural Science Foundation of China (No. 41404004), China Postdoctoral Science Foundation (No. 2014M551790) and Anhui Natural Science Foundation of China (Nos. 1308085QD71 and 1408085QD72). The anonymous reviewer is appreciated for his (her) constructive comments, which helped to improve the manuscript significantly.

\section{References}

Azarbad MR, Mosavi MR (2014) A new method to mitigate multipath error in single-frequency GPS receiver with wavelet transform. GPS Solut 18(2):189-198

Boccia L, Amendola G, Gao S, Chen CC (2014) Quantitative evaluation of multipath rejection capabilities of GNSS antennas. GPS Solut 18(2):199-208 
Brunner FK, Hartinger H, Troyer L (1999) GPS signal diffraction modeling: the stochastic SIGMA- $\Delta$ model. J Geod 73(5):259-267

Dai WJ, Ding XL, Zhu JJ (2008a) Comparing GPS stochastic models based on observation quality indices. Geomat Inf Sci Wuhan Univ 33(7):718-722 (in Chinese)

Dai WJ, Ding XL, Zhu JJ (2008b) Study on multipath effect in structure health monitoring using GPS. J Geod Geodyn 28(1):65-71 (in Chinese)

Dai WJ, Huang DW, Cai CS (2014) Multipath mitigation via component analysis methods for GPS dynamic deformation monitoring. GPS Solut 18(3):417-428

Hartinger H, Brunner FK (1999) Variances of GPS phase observations: the SIGMA- $\varepsilon$ model. GPS Solut 2(4):35-43

Hastaoglu KO, Sanli DU (2011) Accuracy of GPS rapid static positioning: application to Koyulhisar landslide, central Turkey. Surv Rev 43(321):226-240

Liu C, Wang J, Xu CH, Gao JX (2010) Integration of GPS/pseudolites baseline solution based on empirical mode decomposition. Geomat Inf Sci Wuhan Univ 35(8):996-1000 (in Chinese)

Satirapod C (2006) Stochastic models used in static GPS relative positioning. Surv Rev 38(299):379-386

Satirapod C, Rizos C (2005) Multipath mitigation by wavelet analysis for GPS base station applications. Surv Rev 38(295):2-10

Satirapod C, Wang JL, Rizos C (2002) A simplified MINQUE procedure for the estimation of variancecovariance components of GPS observables. Surv Rev 36(286):582-590

Stewart MP, Penna NT, Lichti DD (2005) Investigating the propagation mechanism of unmodelled systematic errors on coordinate time series estimated using least squares. J Geod 79(8):479-489

Townsend B, Fenton P, Van DK, Van NR (1995) L1 carrier phase multipath error reduction using MEDLL technology. In: Proceedings of the 8th international technical meeting of the satellite division of the institute of navigation (ION GPS 1995), Palm Springs, USA, 12-15 September 1995, pp 1539-1544

Tut I, Sanli DU, Erdogan B, Hekimoglu S (2013) Efficiency of BERNESE single baseline rapid static positioning solutions with search strategy. Surv Rev 45(331):296-304

Wang JL, Stewart MP, Tsakiri M (1998) Stochastic modeling for static GPS baseline processing. J Surv Eng 124(4):171-181

Wang JL, Satirapod C, Rizos C (2002) Stochastic assessment of GPS carrier phase measurements for precise static relative positioning. J Geod 76(2):95-104

Wang J, Gao JX, Wang JL, Xu CH (2008) EMD-based GPS baseline solution and validation test. J China Univ Min Technol 18(2):283-287

Wang J, Wang JL, Roberts C (2009) Reducing GPS carrier phase errors with EMD-wavelet for precise static positioning. Surv Rev 41(312):152-161

$\mathrm{Xu}$ GC (2007) GPS theory, algorithms and applications, 2nd edn. Springer, Berlin 